\title{
Effect of Alirocumab on Coronary Atheroma Volume in Japanese Patients With Acute Coronary Syndrome - The ODYSSEY J-IVUS Trial -
}

\author{
Junya Ako, MD, PhD; Kiyoshi Hibi, MD, PhD; Kenichi Tsujita, MD, PhD; \\ Takafumi Hiro, MD, PhD; Yoshihiro Morino, MD, PhD; Ken Kozuma, MD, PhD; \\ Toshiro Shinke, MD, PhD; Hiromasa Otake, MD, PhD; Kiyoko Uno, MD, PhD; \\ Michael J Louie, MD; Yoshiharu Takagi; Katsumi Miyauchi, MD, PhD
}

\begin{abstract}
Background: In patients with acute coronary syndrome (ACS), alirocumab reduced the risk of recurring ischemic events. ODYSSEY J-IVUS assessed the effect of alirocumab on coronary atheroma volume in Japanese patients recently hospitalized with ACS and hypercholesterolemia, using intravascular ultrasound imaging analysis.
\end{abstract}

\begin{abstract}
Methods and Results: Patients $(\mathrm{n}=206)$ who at index ACS diagnosis either had low-density lipoprotein cholesterol (LDL-C) $\geq 2.59 \mathrm{mmol} / \mathrm{L}(\geq 100 \mathrm{mg} / \mathrm{dL}$ ) despite stable statin therapy, or were not on statins with LDL-C levels above target after statin initiation, were randomized (1:1) to alirocumab (75 mg every 2 weeks [Q2W]/up to $150 \mathrm{mg}$ Q2W), or standard of care (SoC; atorvastatin $\geq 10 \mathrm{mg} /$ day or rosuvastatin $\geq 5 \mathrm{mg} /$ day) for 36 weeks. The primary efficacy endpoint (week [W] 36 mean [standard error] percent change in normalized total atheroma volume [TAV] from baseline) was $-3.1(1.0) \%$ with SoC vs. $-4.8(1.0) \%$ with alirocumab (between-group difference: -1.6 [1.4]; $\mathrm{P}=0.23$ ). W36 absolute change from baseline in percent atheroma volume was $-1.3(0.4) \%$ (SoC) and -1.4 (0.4)\% (alirocumab; nominal $\mathrm{P}=0.79$ ). At W36, LDL-C was reduced from baseline by $13.4 \%$ (SoC) vs. $63.9 \%$ (alirocumab; nominal $\mathrm{P}<0.0001$ ). In total, $61.8 \%$ (SoC) and $75.7 \%$ (alirocumab) of patients reported treatment-emergency adverse events.
\end{abstract}

Conclusions: In Japanese patients with ACS and hypercholesterolemia inadequately controlled despite statin therapy, from baseline to W36, a numerically greater percent reduction in normalized TAV was observed with alirocumab vs. SoC, which did not reach statistical significance.

Key Words: Atherosclerosis; Coronary artery disease; Hypercholesterolemia; Intravascular ultrasound; Lipids

$\mathbf{P}$ atients with acute coronary syndrome (ACS) are at very high risk of recurrent cardiovascular $(\mathrm{CV})$ events. ${ }^{1,2}$ Cholesterol management guidelines recommend that these patients receive maximally tolerated statin doses. ${ }^{3-6}$ However, if levels of low-density lipoprotein cholesterol (LDL-C) remain elevated despite maximally tolerated statin therapy, additional lipid-lowering therapy (LLT) should be considered, such as a proprotein conver-

\section{Editorial p 1988}

tase subtilisin/kexin type 9 (PCSK9) inhibitor. ${ }^{7,8}$

In the ODYSSEY OUTCOMES trial, alirocumab was shown to significantly reduce the risk of major adverse $\mathrm{CV}$ events (MACE; hazard ratio [HR], 0.85, 95\% confidence interval $[\mathrm{CI}], 0.78-0.93, \mathrm{P}<0.001)$, and all-cause death

Received May 13, 2019; revised manuscript received July 11, 2019; accepted July 18, 2019; J-STAGE Advance Publication released online August 20, 2019 Time for primary review: 21 days

Department of Cardiovascular Medicine, Kitasato University School of Medicine, Sagamihara (J.A.); Division of Cardiology, Yokohama City University Medical Center, Yokohama (K.H.); Department of Cardiovascular Medicine, Graduate School of Medical Sciences, Kumamoto University, Kumamoto (K.T.); Division of Cardiology, Department of Medicine, Nihon University School of Medicine, Tokyo (T.H.); Division of Cardiology, Department of Internal Medicine, Iwate Medical University, Morioka (Y.M.); Division of Cardiology, Teikyo University Hospital, Tokyo (K.K.); Division of Cardiovascular Medicine, Department of Internal Medicine, Showa University School of Medicine, Tokyo (T.S.); Division of Cardiovascular Medicine, Department of Internal Medicine, Kobe University Graduate School of Medicine, Kobe (H.O.); Cardiovascular Medical (K.U.), Clinical Sciences and Operations (Y.T.), Sanofi, Tokyo, Japan; Regeneron Pharmaceuticals, Inc., Tarrytown, NY (M.J.L.), USA; and Department of Cardiovascular Medicine, Juntendo University Graduate School of Medicine, Tokyo (K.M.), Japan

Clinical trial registration: URL: https://www.clinicaltrials.gov Unique identifier: NCT02984982

Mailing address: Junya Ako, MD, PhD, Department of Cardiovascular Medicine, Kitasato University School of Medicine, 1-15-1 Kitasato, Minami-ku, Sagamihara 252-0374, Japan. E-mail: jako@kitasato-u.ac.jp

ISSN-1346-9843 All rights are reserved to the Japanese Circulation Society. For permissions, please e-mail: cj@j-circ.or.jp 
(HR, 0.85; 95\% CI, 0.73-0.98) with nominally significant P-value vs. placebo in a population of patients with recent (within 1-12 months) ACS.9 No interaction was observed between treatment effect and geographic region (interaction P-value $=0.40) .{ }^{9}$ The use of intravascular ultrasound (IVUS) to evaluate atheroma volume is a globally established method to evaluate the CV effect of LLT.10 In a metaanalysis $(n=4,137)$, a direct relationship was shown between the burden of coronary atherosclerosis as defined by IVUS, its progression, and adverse CV events. ${ }^{11}$

The present study (ODYSSEY J-IVUS; NCT02984982) assessed the effect of alirocumab on coronary atherosclerotic plaque in Japanese patients with recent hospitalization for ACS who, despite statin therapy, did not achieve the recommended LDL-C levels as defined by the Japan Atherosclerotic Society. ${ }^{6}$

\section{Methods}

ODYSSEY J-IVUS was a Phase IV, 36-week, open-label, randomized, blinded IVUS analysis, parallel-group, multicenter study of Japanese patients who had recently been hospitalized for ACS and who had hypercholesterolemia inadequately controlled despite statin therapy. The study was conducted at 40 sites in Japan; the first patient was enrolled in November 2016 and the last in November 2017. The study design and methods have been published previously. ${ }^{12}$

The study was conducted in accordance with the ethical principles derived from the Declaration of Helsinki and the International Conference of Harmonization guidelines for Good Clinical Practice, and with all applicable laws, rules, and regulations. The study protocol was approved by the relevant institutional review boards of participating centers, and all participating patients provided written informed consent.

\section{Patients}

The study included 206 patients (aged $\geq 20$ years) who had been hospitalized for ACS, had LDL-C $\geq 2.59 \mathrm{mmol} / \mathrm{L}$ $(\geq 100 \mathrm{mg} / \mathrm{dL})$ at ACS diagnosis, had undergone IVUS imaging as part of usual clinical practice in Japan, and had an analyzable IVUS image of the culprit or non-culprit vessel with $\geq 50 \%$ angiographic stenosis of the culprit vessel within 1 week after ACS onset. ACS was defined as STelevation myocardial infarction (STEMI), non-STEMI, and unstable angina (Supplementary Table 1). Eligible patients with LDL-C $\geq 2.59 \mathrm{mmol} / \mathrm{L}$ ( $\geq 100 \mathrm{mg} / \mathrm{dL}$ ) who had been on any statin therapy at ACS onset received atorvastatin $\geq 10 \mathrm{mg}$ /day or rosuvastatin $\geq 5 \mathrm{mg} /$ day (if not already on these), based on the investigator's judgement. Eligible patients with LDL-C $\geq 2.59 \mathrm{mmol} / \mathrm{L}(\geq 100 \mathrm{mg} / \mathrm{dL})$ not taking a statin at ACS diagnosis were started on atorvastatin $10 \mathrm{mg}$ /day or rosuvastatin $5 \mathrm{mg}$ /day immediately after diagnosis, and could enter the study if their LDL-C level was $\geq 2.59 \mathrm{mmol} / \mathrm{L}(\geq 100 \mathrm{mg} / \mathrm{dL}$; or $\geq 1.81 \mathrm{mmol} / \mathrm{L}[\geq 70 \mathrm{mg} / \mathrm{dL}]$, if the investigator deemed it appropriate) after 2-4 weeks. A full list of study inclusion and exclusion criteria is presented in Supplementary Table 1.

\section{Study Design}

ODYSSEY J-IVUS consisted of a 36-week open-label treatment period, which started within 4 weeks after ACS diagnosis with or without revascularization and also included post-treatment IVUS imaging at Week 36 (end of treatment \pm 2 weeks; Supplementary Figure 1). Following the treatment period there was a safety follow-up period (off treatment) of 21 days.

Eligible patients were randomized (1:1) by permutedblock design to receive either alirocumab or standard-ofcare (SoC). Randomization was stratified by previous use of statin therapy at the index ACS onset. Patients in the alirocumab group received alirocumab $75 \mathrm{mg}$ every 2 weeks (Q2 W) in addition to stable dose background statin therapy with/without other LLTs (Supplementary Figure 1). At Week 14, the study allowed alirocumab dose increase to $150 \mathrm{mg}$ Q2 W if Week $12 \mathrm{LDL}-\mathrm{C}$ was $\geq 2.59 \mathrm{mmol} / \mathrm{L}$ $(\geq 100 \mathrm{mg} / \mathrm{dL})$. Patients in the SoC group received stable dose statin therapy, with optional dose adjustment (within the range approved by health authority). Data for statin dose changes were not available. In patients receiving statin monotherapy in the SoC arm, non-statin, non-PCSK9 inhibitor LLTs could be added by investigators if LDL-C goal $<2.59 \mathrm{mmol} / \mathrm{L}(<100 \mathrm{mg} / \mathrm{dL})$ could not be achieved; adjustments could be made after randomization during the treatment phase. Baseline IVUS images were obtained from culprit or non-culprit vessels as the longest possible length with or without signs of atherosclerosis. Using digitized images, the central laboratory, blinded to the patient's background and study treatment, selected a target vessel segment $>10 \mathrm{~mm}$ for serial analysis on the basis of IVUS land-marks (side branches or stent) with preference for the culprit vessel. The IVUS imaging of a target vessel was then performed at Week 36. Investigators were recommended to use the same IVUS imaging system for both the baseline and follow-up with auto-pullback speed of $0.5 \mathrm{~mm} / \mathrm{s}$ [40 MHz or $60 \mathrm{MHz}$ commercially available IVUS imaging catheter (Boston Scientific, Natick, MA, USA; Terumo Corp., Tokyo, Japan)].

\section{Study Endpoints}

On-site visits took place during the treatment period at Weeks $0,4,12,24$, and 36. Blood sampling was performed in the morning under fasting conditions, which was defined as an overnight fast of $\geq 8 \mathrm{~h}$.

The primary efficacy endpoint was the percent change in normalized total atheroma volume (TAV) from baseline to Week 36, defined as follows: Normalized TAV (Week 36)Normalized TAV (baseline)/Normalized TAV (baseline) $\times$ 100. Normalized TAVs at baseline and Week 36 were obtained prior to randomization and after $\geq 24$ weeks of treatment, respectively. Normalized TAV was calculated as previously described; 12 briefly:

Normalized TAV $\left(\mathrm{mm}^{3}\right)=\Sigma($ EEMcsA - LumencsA $) \delta \times \mathrm{C} / \mathrm{n}$

where EEMcsa is the cross-sectional area inside the external elastic membrane border, Lumencsa the cross-sectional area inside the lumen border, $\Sigma$ the summation over all analyzed frames, $\delta$ the pre-determined intervals between each image $(0.5 \mathrm{~mm}), \mathrm{n}$ the number of analyzed frames per patient, and $\mathrm{C}$ the median of the number of analyzed frames in patients with available baseline TAV within the randomized population. EEMcsa and LumencsA were measured by the central laboratory, blinded to study treatment groups.

The key secondary efficacy endpoint was the absolute change in percent atheroma volume (PAV) from baseline to Week 36. PAV was calculated as previously described: ${ }^{6}$

PAV $(\%)=(\Sigma($ EEMcsA - LumencsA $) / \Sigma$ EEMcsA $) \times 100$ 


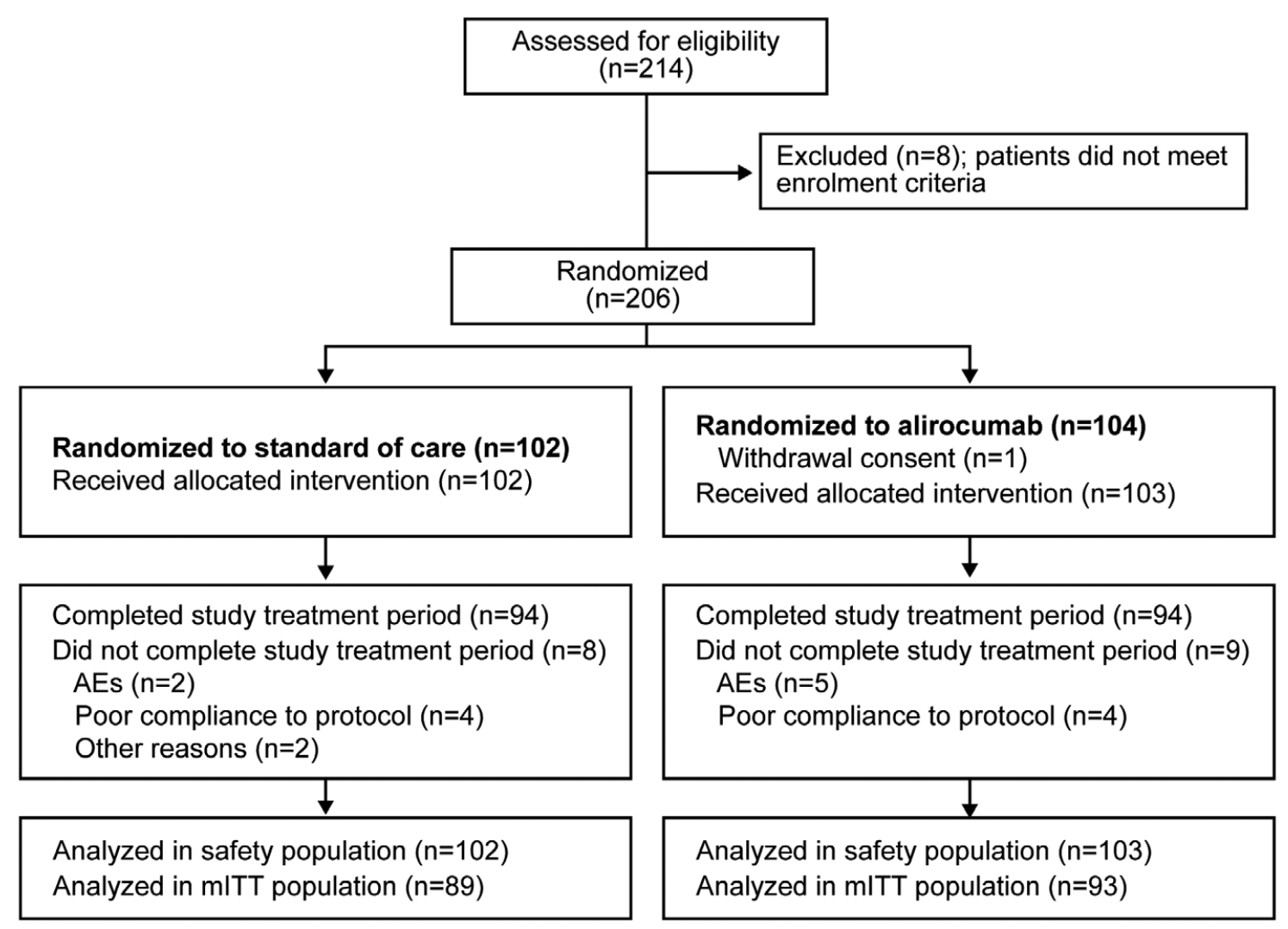

Figure. Patient flow diagram for J-IVUS. AE, adverse event; mITT, modified intention-to-treat.

Other secondary endpoints are listed in Supplementary Table 2. All lipids were measured by a central laboratory. LDL-C was calculated using the Friedewald formula. ${ }^{13}$ If triglyceride values exceeded $4.5 \mathrm{mmol} / \mathrm{L}(400 \mathrm{mg} / \mathrm{dL})$, the central laboratory automatically measured LDL-C directly, using the $\beta$-quantification method.

Safety parameters, including adverse events (AEs), laboratory data, and vital signs were assessed throughout the study.

\section{Study Populations}

The randomized population included patients who were allocated to treatment and recorded in the registration center database, regardless of whether study drug was received.

The primary efficacy analysis population was the modified intent-to-treat (mITT) population, including randomized patients who took $\geq 1$ dose or part of a dose of study drug and had an available value of normalized TAV before randomization and after 24 weeks of treatment.

The safety population was defined as the randomized population who received $\geq 1$ dose or part of a dose of the study drug and analyzed according to the treatment received. Randomized patients for whom it was unclear whether they took the study drug were included in the safety population.

\section{Statistical Analysis}

The sample size was calculated using the results of the
eZEtimibe Ultrasound Study (ZEUS) ${ }^{\mathbf{1 4}}$ and the Plaque Regression With Cholesterol Absorption Inhibitor or Synthesis Inhibitor or Synthesis Inhibitor Evaluated by Intravascular Ultrasound (PRECISE-IVUS) study. ${ }^{15}$ In the PRECISE-IVUS study, percent change from baseline in normalized TAV for patients with ACS was $-10.2 \%$ for atorvastatin+ezetimibe, and $-1.3 \%$ for atorvastatin only. ${ }^{14}$ In the ZEUS study, standard deviation (SD) of percent change in TAV was $12.6 \%{ }^{15}$ Thus, it was assumed that the difference in percent change in normalized TAV from baseline between alirocumab and SoC arms would be $8 \%$, and the common SD of percent change in normalized TAV would be $15 \%$. Therefore, a sample size of 150 patients ( $\mathrm{n}=75$ [alirocumab group] and $\mathrm{n}=75$ [SoC group]) would have $90 \%$ power to detect the treatment difference with a 2 -sided significance level of 5\%. Assuming that the proportion of patients with a non-evaluable primary endpoint would be $25 \%$, it was considered that 200 patients $(n=100$ in both treatment groups) would be needed.

As previously reported, the primary efficacy endpoint was analyzed in the mITT population using an analysis of covariance model with treatment arm and randomization strata (statin at ACS onset) as fixed effects, and the baseline normalized TAV as covariate. ${ }^{\mathbf{1 2}}$ Briefly, the alirocumab arm was compared with the SoC arm at the 2-sided 0.05 level for superiority, and least-squares means at Week 36 for each treatment arm were calculated.

For the key secondary efficacy endpoint and the other secondary efficacy endpoints, descriptive summaries and 
analyses were performed in the mITT population. ${ }^{12}$ Statistical significance of the primary efficacy endpoint at the 0.05 alpha level was required before sequentially testing key secondary efficacy endpoints at the 0.05 level.

Safety analyses were descriptive, based on the safety population. The safety analysis focused on the treatmentemergent AE (TEAE) period (defined as the time from randomization to the last administration +21 days or end of study, whichever came first).

\section{Results}

\section{Patients' Characteristics}

A total of 206 patients were randomized 1:1 to alirocumab $(n=104)$ or SoC $(n=102$; Figure). Baseline characteristics and lipid levels were similar regardless of allocated treatment (Table 1). Mean baseline LDL-C was 2.48 and $2.54 \mathrm{mmol} / \mathrm{L}$ ( 95.7 and $97.9 \mathrm{mg} / \mathrm{dL}$ ) in the $\mathrm{SoC}$ and alirocumab groups, respectively. At baseline, mean (SD) normalized TAV was $103.3(50.3) \mathrm{mm}^{3}$ in the SoC group and $110.3(56.1) \mathrm{mm}^{3}$ in the alirocumab group. In the alirocumab group, all patients in the mITT population received alirocumab over
24 weeks. Most patients were using background statins at the lowest available doses at baseline (atorvastatin $10 \mathrm{mg}$ or rosuvastatin $5 \mathrm{mg}$ ), with a few patients using higher doses (Table 1). Background ezetimibe was used by $7.9 \%$ and $7.5 \%$ of patients in the SoC and alirocumab groups, respectively.

\section{Efficacy}

The percentage change (standard error [SE]) from baseline to Week 36 in normalized TAV was $-3.1(1.0) \%$ in the SoC group and $-4.8(1.0) \%$ in the alirocumab group, resulting in $\mathrm{a}-1.6(1.4) \%$ mean difference $(\mathrm{P}=0.23$; mITT population; Table 2).

The mean (SE) absolute change from baseline to Week 36 in PAV was $-1.3(0.4) \%$ and $-1.4(0.4) \%$ in the SoC and alirocumab groups, respectively (mean difference: $-0.2 \%$; nominal $\mathrm{P}=0.79$; Table 2). No significant difference between the SoC and alirocumab groups was observed for other prespecified secondary efficacy endpoints related to plaque volume, except for absolute change from baseline to Week 36 in TAV, which was further decreased in the alirocumab group $\left(8.3 \mathrm{~mm}^{3}\right.$ reduction) compared with the SoC group

\begin{tabular}{|c|c|c|}
\hline & $\begin{array}{l}\text { Standard of care } \\
(n=89)\end{array}$ & $\begin{array}{c}\text { Alirocumab } \\
(n=93)\end{array}$ \\
\hline Age, years, mean (SD) & $60.5(11.6)$ & $61.8(10.2)$ \\
\hline Sex, male, $\mathrm{n}(\%)$ & $72(80.9)$ & $74(79.6)$ \\
\hline BMI, kg/m², mean (SD) & $25.0(3.6)$ & $25.2(3.8)$ \\
\hline CAD prior to the index ACS diagnosis, $n(\%)$ & $10(11.2)$ & $12(12.9)$ \\
\hline Ischemic stroke, $\mathrm{n}(\%)$ & $3(3.4)$ & $5(5.4)$ \\
\hline PAD, n (\%) & 0 & $1(1.1)$ \\
\hline Hypertension, n (\%) & $63(70.8)$ & $64(68.8)$ \\
\hline Diabetes, n (\%) & $31(34.8)$ & $27(29.0)$ \\
\hline Type 1 diabetes & $1(1.1)$ & 0 \\
\hline Type 2 diabetes & $30(33.7)$ & $27(29.0)$ \\
\hline CKD, n (\%) & $10(11.2)$ & $9(9.7)$ \\
\hline Impaired glucose tolerance, $\mathrm{n}(\%)$ & $11(12.4)$ & $6(6.5)$ \\
\hline $\mathrm{HeFH}, \mathrm{n}(\%)$ & $4(4.5)$ & $5(5.4)$ \\
\hline Statin therapy at ACS onset, $\mathrm{n}(\%)$ & $31(34.8)$ & $34(36.6)$ \\
\hline Any statin at randomization, $\mathrm{n}(\%)$ & $89(100)$ & $93(100)$ \\
\hline Atorvastatin & $49(55.1)$ & $56(60.2)$ \\
\hline $10 \mathrm{mg}$ & $46(51.7)$ & $51(54.8)$ \\
\hline $20 \mathrm{mg}$ & $3(3.4)$ & $5(5.4)$ \\
\hline Rosuvastatin & $40(44.9)$ & $37(39.8)$ \\
\hline $5 \mathrm{mg}$ & $39(43.8)$ & $35(37.6)$ \\
\hline $10 \mathrm{mg}$ & 0 & $2(2.2)$ \\
\hline $20 \mathrm{mg}$ & $1(1.1)$ & 0 \\
\hline Any LLT (other than statin), $\mathrm{n}(\%)$ & $13(14.6)$ & $11(11.8)$ \\
\hline Ezetimibe & $7(7.9)$ & $7(7.5)$ \\
\hline Fibrate & 0 & 0 \\
\hline Other & $6(6.7)$ & $4(4.3)$ \\
\hline Aspirin or oral ADP receptor antagonist, $\mathrm{n}(\%)$ & $89(100)$ & $93(100)$ \\
\hline Aspirin & $89(100)$ & $92(98.9)$ \\
\hline Oral ADP receptor antagonists (except aspirin) & $88(98.9)$ & $91(97.8)$ \\
\hline Prasugrel & $68(76.4)$ & $72(77.4)$ \\
\hline Clopidogrel & $20(22.5)$ & $18(19.4)$ \\
\hline Ticlopidine & 0 & $1(1.1)$ \\
\hline Ticagrelor & 0 & 0 \\
\hline
\end{tabular}


Analyzed vessel length, $\mathrm{mm}$

Location of analyzed IVUS imaging vessels, $n$ (\%)

RCA

LAD

LCX

Other

Normalized TAV, $\mathrm{mm}^{3}$, mean (SD)

PAV, \%, mean (SD)

EEM volume, $\mathrm{mm}^{3}$, mean (SD)

Lumen volume, $\mathrm{mm}^{3}$, mean (SD)

$\mathrm{TAV}, \mathrm{mm}^{3}$, mean (SD)

Index ACS subtype, $\mathrm{n}(\%)$

STEMI

NSTEMI

Unstable angina

Lipids, mean (SD)

LDL-C, calculated, $\mathrm{mmol} / \mathrm{L}[\mathrm{mg} / \mathrm{dL}]$

Apo B, g/L [mg/dL]

Non-HDL-C, mmol/L [mg/dL]

Total cholesterol, $\mathrm{mmol} / \mathrm{L}[\mathrm{mg} / \mathrm{dL}]$

Lp(a), median (Q1: Q3), g/L [mg/dL]

$\mathrm{HDL}-\mathrm{C}, \mathrm{mmol} / \mathrm{L}$ [mg/dL]

Fasting TGs, median (Q1: Q3), $\mathrm{mmol} / \mathrm{L}$ [mg/dL]

Apo A1, g/L [mg/dL]

\section{Standard of care \\ $(n=89)$ \\ $20.2(9.5)$}

$30(33.7)$

$46(51.7)$

$10(11.2)$

$3(3.4)$

$103.3(50.3)$

$44.0(11.6)$

282.3 (171.8)

150.7 (83.5)

$131.6(97.9)$

$49(55.1)$

$15(16.9)$

$25(28.1)$

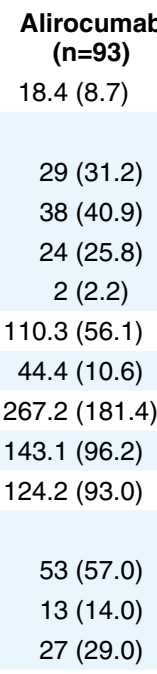

$2.54(0.60)$ [97.9 (23.2)]

$0.91(0.18)[90.9(18.4)]$

$3.21(0.65)[124.1(25.3)]$

$4.35(0.73)$ [168.0 (28.3)]

$0.18(0.1: 0.4)$ [18.0 (10.0:43.0)]

$1.14(0.26)[43.9(10.0)]$

$1.31(1.07: 1.86)$ [115.5 (94.5:164.5)]

$1.24(0.23)[124.1(22.7)]$

ACS, acute coronary syndrome; ADP, adenosine diphosphate; Apo, apolipoprotein; BMI, body mass index; CAD, coronary artery disease; CKD, chronic kidney disease; EEM, external elastic membrane; HDL-C, high-density lipoprotein; HeFH, heterozygous familial hypercholesterolemia; LAD, left anterior descending coronary artery; LCX, left circumflex coronary artery; LDL-C, low-density lipoprotein cholesterol; LLT, lipid-lowering therapy; Lp(a), lipoprotein (a); mITT, modified intention-to-treat; NSTEMI, non-ST-elevation myocardial infarction; PAD, peripheral arterial disease; PAV, percent atheroma volume; RCA, right coronary artery; SD, standard deviation; STEMI, ST-elevation myocardial infarction; TAV, total atheroma volume; TG, triglyceride.

\begin{tabular}{|c|c|c|c|}
\hline & $\begin{array}{c}\text { Standard of care } \\
(n=89)\end{array}$ & $\begin{array}{c}\text { Alirocumab } \\
(n=93)\end{array}$ & P-value* \\
\hline \multicolumn{4}{|l|}{ Primary endpoint } \\
\hline Percentage change from baseline in normalized TAV, LS mean (SE) & $-3.1(1.0)$ & $-4.8(1.0)$ & \\
\hline LS mean difference (SE) & \multicolumn{2}{|c|}{$-1.6(1.4)$} & 0.2279 \\
\hline \multicolumn{4}{|l|}{ Key secondary endpoint } \\
\hline PAV absolute change from baseline, $\%$, LS mean (SE) & $-1.3(0.4)$ & $-1.4(0.4)$ & \\
\hline LS mean difference (SE) & \multicolumn{2}{|c|}{$-0.2(0.5)$} & $0.7898^{\dagger}$ \\
\hline \multicolumn{4}{|l|}{ Other secondary endpoints } \\
\hline Normalized TAV absolute change from baseline, $\mathrm{mm}^{3}$, LS mean (SE) & $-4.7(1.0)$ & $-5.8(1.0)$ & \\
\hline LS mean difference (SE) & \multicolumn{2}{|c|}{$-1.0(1.4)$} & $0.4687^{\dagger}$ \\
\hline EEM volume absolute change from baseline, $\mathrm{mm}^{3}$, estimate for adjusted mean (SE) & $-8.2(1.9)$ & $-10.0(1.8)$ & \\
\hline Estimate for adjusted mean difference (SE) & \multicolumn{2}{|c|}{$-1.8(2.6)$} & $0.49466^{\dagger}$ \\
\hline EEM volume percentage change from baseline, \%, estimate for adjusted mean (SE) & $-0.9(0.9)$ & $-3.2(0.9)$ & \\
\hline Estimate for adjusted mean difference (SE) & \multicolumn{2}{|c|}{$-2.3(1.3)$} & $0.0731^{\dagger}$ \\
\hline Lumen volume absolute change from baseline, $\mathrm{mm}^{3}$, estimate for adjusted mean (SE) & $-1.3(1.4)$ & $-0.9(1.4)$ & \\
\hline Estimate for adjusted mean difference (SE) & \multicolumn{2}{|c|}{$0.3(1.9)$} & $0.8695^{\dagger}$ \\
\hline Lumen volume percentage change from baseline, $\%$, estimate for adjusted mean (SE) & $1.2(1.3)$ & $-0.9(1.2)$ & \\
\hline Estimate for adjusted mean difference (SE) & \multicolumn{2}{|c|}{$-2.1(1.8)$} & $0.2437^{\dagger}$ \\
\hline TAV absolute change from baseline, $\mathrm{mm}^{3}$, estimate for adjusted mean (SE) & $-5.1(1.0)$ & $-8.3(1.0)$ & \\
\hline Estimate for adjusted mean difference (SE) & \multicolumn{2}{|c|}{$-3.3(1.4)$} & $0.0208^{\dagger}$ \\
\hline TAV percentage change from baseline, \%, estimate for adjusted mean (SE) & $-3.0(1.0)$ & $-5.4(1.0)$ & \\
\hline Estimate for adjusted mean difference (SE) & \multicolumn{2}{|c|}{$-2.4(1.4)$} & $0.0866^{\dagger}$ \\
\hline
\end{tabular}

${ }^{*}$ Alirocumab vs. standard of care. ${ }^{\dagger}$ P-value is nominal. LS, least-squares; SE, standard error. Other abbreviations as in Table 1. 
Table 3. Absolute and Percentage Change From Baseline in LDL-C at Weeks 12 and 36 , and Other Lipids at Week 36 in the mITT Population

\begin{tabular}{|c|c|c|c|}
\hline & $\begin{array}{l}\text { Standard of care } \\
(n=89)\end{array}$ & $\begin{array}{l}\text { Alirocumab } \\
(n=93)\end{array}$ & P-value \\
\hline \multicolumn{4}{|l|}{ Week 12} \\
\hline $\begin{array}{l}\text { LDL-C (calculated) absolute change from baseline, } \\
\mathrm{mmol} / \mathrm{L}[\mathrm{mg} / \mathrm{dL}], \mathrm{LS} \text { mean (SE) }\end{array}$ & $-0.25(0.04)[-9.6(1.7)]$ & $-1.62(0.04)[-62.4(1.6)]$ & \\
\hline LS mean difference (SE) & \multicolumn{2}{|c|}{$-1.37(0.06)[-52.8(2.4)]$} & $<0.0001$ \\
\hline $\begin{array}{l}\text { LDL-C (calculated) percentage change from baseline, } \% \text {, } \\
\text { LS mean (SE) }\end{array}$ & $-7.6(1.9)$ & $-64.5(1.8)$ & \\
\hline LS mean difference (SE) & \multicolumn{2}{|c|}{$-57.0(2.6)$} & $<0.0001$ \\
\hline \multicolumn{4}{|l|}{ Week 36} \\
\hline $\begin{array}{l}\text { LDL-C (calculated) absolute change from baseline, } \\
\mathrm{mmol} / \mathrm{L} \text { [mg/dL], LS mean (SE) }\end{array}$ & $-0.40(0.05)[-15.5(1.8)]$ & $-1.64(0.05)[-63.2(1.8)]$ & \\
\hline LS mean difference (SE) & \multicolumn{2}{|c|}{$-1.24(0.07)[-47.8(2.5)]$} & $<0.0001$ \\
\hline $\begin{array}{l}\text { LDL-C (calculated) percentage change from baseline, } \% \text {, } \\
\text { LS mean (SE) }\end{array}$ & $-13.4(2.0)$ & $-63.9(1.9)$ & \\
\hline LS mean difference (SE) & \multicolumn{2}{|c|}{$-50.5(2.8)$} & $<0.0001$ \\
\hline $\begin{array}{l}\text { Apo B absolute change from baseline, } \mathrm{g} / \mathrm{L}[\mathrm{mg} / \mathrm{dL}] \\
\text { LS mean (SE) }\end{array}$ & $-0.17(0.01)[-16.8(1.4)]$ & $-0.51(0.01)[-51.0(1.3)]$ & \\
\hline LS mean difference (SE) & \multicolumn{2}{|c|}{$-0.34(0.02)[-34.2(1.9)]$} & $<0.0001$ \\
\hline Apo B percentage change from baseline, $\%$, LS mean (SE) & $-16.6(1.6)$ & $-55.1(1.5)$ & \\
\hline LS mean difference (SE) & \multicolumn{2}{|c|}{$-38.5(2.2)$} & $<0.0001$ \\
\hline $\begin{array}{l}\text { Non-HDL-C absolute change from baseline, } \mathrm{mmol} / \mathrm{L}[\mathrm{mg} / \mathrm{dL}] \text {, } \\
\text { LS mean (SE) }\end{array}$ & $-0.53(0.05)[-20.3(2.0)]$ & $-1.79(0.05)[-69.2(2.0)]$ & \\
\hline LS mean difference (SE) & \multicolumn{2}{|c|}{$-1.26(0.07)[-48.9(2.8)]$} & $<0.0001$ \\
\hline $\begin{array}{l}\text { Non-HDL-C percentage change from baseline, } \% \text {, } \\
\text { LS mean (SE) }\end{array}$ & $-14.1(1.7)$ & $-54.5(1.7)$ & \\
\hline LS mean difference (SE) & \multicolumn{2}{|c|}{$-40.5(2.4)$} & $<0.0001$ \\
\hline $\begin{array}{l}\text { Total cholesterol absolute change from baseline, } \\
\mathrm{mmol} / \mathrm{L}[\mathrm{mg} / \mathrm{dL}], \mathrm{LS} \text { mean (SE) }\end{array}$ & $-0.39(0.06)[-15.2(2.3)]$ & $-1.60(0.06)[-61.7(2.2)]$ & \\
\hline LS mean difference (SE) & \multicolumn{2}{|c|}{$-1.20(0.08)[-46.5(3.2)]$} & $<0.0001$ \\
\hline $\begin{array}{l}\text { Total cholesterol percentage change from baseline, } \% \text {, } \\
\text { LS mean (SE) }\end{array}$ & $-7.6(1.4)$ & $-35.4(1.3)$ & \\
\hline LS mean difference (SE) & \multicolumn{2}{|c|}{$-27.8(1.9)$} & $<0.0001$ \\
\hline $\begin{array}{l}\text { Lp(a) absolute change from baseline, } g / L \text { [mg/dL], } \\
\text { estimate for adjusted mean (SE) }\end{array}$ & $-0.10(0.01)[-10.3(0.5)]$ & $-0.16(0.01)[-15.5(0.5)]$ & \\
\hline Estimate for adjusted mean difference (SE) & \multicolumn{2}{|c|}{$-0.05(0.01)[-5.2(0.7)]$} & $<0.0001$ \\
\hline $\begin{array}{l}\mathrm{Lp}(\mathrm{a}) \text { percentage change from baseline, \%, estimate for } \\
\text { adjusted mean (SE) }\end{array}$ & $-17.2(2.6)$ & $-55.8(2.5)$ & \\
\hline Estimate for adjusted mean difference (SE) & \multicolumn{2}{|c|}{$-38.5(3.6)$} & $<0.0001$ \\
\hline $\begin{array}{l}\text { HDL-C absolute change from baseline, } \mathrm{mmol} / \mathrm{L}[\mathrm{mg} / \mathrm{dL}] \\
\text { LS mean (SE) }\end{array}$ & $0.12(0.02)[4.7(0.9)]$ & $0.21(0.02)[8.1(0.9)]$ & \\
\hline LS mean difference (SE) & \multicolumn{2}{|c|}{$0.09(0.03)[3.4(1.3)]$} & 0.0090 \\
\hline HDL-C percentage change from baseline, $\%$, LS mean (SE) & $12.2(2.3)$ & $21.0(2.3)$ & \\
\hline LS mean difference (SE) & \multicolumn{2}{|c|}{$8.9(3.2)$} & 0.0066 \\
\hline $\begin{array}{l}\text { Fasting TGs absolute change from baseline, } \mathrm{mmol} / \mathrm{L}[\mathrm{mg} / \mathrm{dL}] \\
\text { combined estimate for adjusted mean (SE) }\end{array}$ & $-0.30(0.05)[-26.2(4.7)]$ & $-0.40(0.05)[-35.3(4.5)]$ & \\
\hline Combined estimate for adjusted mean difference (SE) & \multicolumn{2}{|c|}{$-0.10(0.07)[-9.1(6.4)]$} & 0.1563 \\
\hline $\begin{array}{l}\text { Fasting TGs percentage from baseline, \%, combined } \\
\text { estimate for adjusted mean (SE) }\end{array}$ & $-8.9(3.6)$ & $-18.4(3.5)$ & \\
\hline Combined estimate for adjusted mean difference (SE) & \multicolumn{2}{|c|}{$-9.5(5.1)$} & 0.0594 \\
\hline $\begin{array}{l}\text { Apo A1 absolute change from baseline, } \mathrm{g} / \mathrm{L}[\mathrm{mg} / \mathrm{dL}] \\
\text { LS mean (SE) }\end{array}$ & $0.04(0.02)[3.8(1.9)]$ & $0.12(0.02)[12.0(1.9)]$ & \\
\hline LS mean difference (SE) & \multicolumn{2}{|c|}{$0.08(0.03)[8.2(2.7)]$} & 0.0026 \\
\hline Apo A1 percentage change from baseline, \%, LS mean (SE) & $4.6(1.6)$ & $11.8(1.6)$ & \\
\hline LS mean difference (SE) & \multicolumn{2}{|c|}{$7.1(2.3)$} & 0.0019 \\
\hline
\end{tabular}

*Nominal P-value for alirocumab vs. standard of care. Abbreviations as in Tables 1,2. 


\begin{tabular}{|c|c|c|}
\hline n (\%) & $\begin{array}{l}\text { Standard of care } \\
(n=102)\end{array}$ & $\begin{array}{c}\text { Alirocumab } \\
(n=103)\end{array}$ \\
\hline Any TEAE & $63(61.8)$ & $78(75.7)$ \\
\hline Treatment-emergent SAEs & $17(16.7)$ & $19(18.4)$ \\
\hline TEAEs leading to death & $1(1.0)$ & 0 \\
\hline TEAEs leading to permanent treatment discontinuation & $2(2.0)$ & $4(3.9)$ \\
\hline Treatment-emergent CV events confirmed by adjudication & $4(3.9)$ & $8(7.8)$ \\
\hline Myocardial infarction & $3(2.9)$ & $2(1.9)$ \\
\hline Ischemic stroke & 0 & $2(1.9)$ \\
\hline Ischemia-driven coronary revascularization procedure & $2(2.0)$ & $4(3.9)$ \\
\hline \multicolumn{3}{|l|}{ TEAEs (preferred term level) in $\geq 2 \%$ of patients in any group } \\
\hline Nasopharyngitis & $15(14.7)$ & $27(26.2)$ \\
\hline Injection-site reaction & 0 & $7(6.8)$ \\
\hline Back pain & 0 & $6(5.8)$ \\
\hline Angina pectoris & $2(2.0)$ & $5(4.9)$ \\
\hline Coronary artery stenosis & $2(2.0)$ & $5(4.9)$ \\
\hline Epistaxis & $2(2.0)$ & $5(4.9)$ \\
\hline Insomnia & 0 & $5(4.9)$ \\
\hline Constipation & 0 & $4(3.9)$ \\
\hline Cough & $2(2.0)$ & $4(3.9)$ \\
\hline Dermatitis contact & $1(1.0)$ & $4(3.9)$ \\
\hline Fall & $1(1.0)$ & $4(3.9)$ \\
\hline Headache & $3(2.9)$ & $4(3.9)$ \\
\hline Hyperuricemia & 0 & $4(3.9)$ \\
\hline Urticaria & $1(1.0)$ & $4(3.9)$ \\
\hline Contusion & $1(1.0)$ & $3(2.9)$ \\
\hline Gastroenteritis & 0 & $3(2.9)$ \\
\hline Hypertension & $3(2.9)$ & $3(2.9)$ \\
\hline Palpitations & 0 & $3(2.9)$ \\
\hline Wound & $1(1.0)$ & $3(2.9)$ \\
\hline Myocardial ischemia & $5(4.9)$ & $2(1.9)$ \\
\hline Diarrhea & $4(3.9)$ & $1(1.0)$ \\
\hline Pneumonia & $4(3.9)$ & $1(1.0)$ \\
\hline Prinzmetal angina & $3(2.9)$ & $1(1.0)$ \\
\hline Type 2 diabetes mellitus & $3(2.9)$ & 0 \\
\hline
\end{tabular}

$\mathrm{CV}$, cardiovascular; SAE, serious adverse event; TEAE, treatment-emergent adverse event.

$\left(5.1 \mathrm{~mm}^{3}\right.$ reduction; $-3.3 \mathrm{~mm}^{3}$ estimate for adjusted mean difference; nominal $\mathrm{P}=0.02$; Table 2).

At Week 12, alirocumab reduced LDL-C from baseline by $64.5 \%$ vs. $7.6 \%$ with SoC (nominal $\mathrm{P}<0.0001$ vs. SoC; Table 3). No patients in the alirocumab arm had their dose increased at Week 12. Alirocumab treatment resulted in a greater mean percent change from baseline to Week 36 (63.9\% reduction) compared with the SoC group (13.4\% reduction; mean difference: $50.5 \%$ reduction vs. SoC; nominal $\mathrm{P}<0.0001$; Table 3). LDL-C $<1.81 \mathrm{mmol} / \mathrm{L}$ $(<70 \mathrm{mg} / \mathrm{dL})$ at Week 36 was achieved by $32.6 \%$ of patients in the $\mathrm{SoC}$ group and $95.7 \%$ in the alirocumab group (nominal $\mathrm{P}<0.0001$ ). Further lipid parameter results are shown in Table 3.

The results of a subgroup analysis of percent change from baseline to Week 36 in normalized TAV according to baseline subgroups are presented in Supplementary Figure 2.

\section{Safety}

The frequency of TEAEs was $61.8 \%$ in the SoC group and $75.7 \%$ in the alirocumab group (Table 4). There was 1 death in the SoC arm and no deaths in the alirocumab arm; the patient in the SoC arm died during the TEAE period from sepsis, ACS, and cardiac failure.

The TEAEs occurring in $\geq 2 \%$ of patients were reported at generally similar frequencies in the $\mathrm{SoC}$ and alirocumab groups; nasopharyngitis (SoC: 14.7\%; alirocumab: 26.2\%) was the most common TEAE. In total, 7 (6.8\%) patients in the alirocumab group and none in the SoC group experienced $\geq 1$ local injection-site reaction. Most of these $(n=6)$ were classified as mild in intensity $(n=1$ moderate intensity), and all patients continued to receive study treatment.

\section{Discussion}

ODYSSEY J-IVUS aimed to assess the effect of the PCSK9 inhibitor alirocumab on coronary atheroma volume in Japanese ACS patients. Alirocumab treatment over 36 weeks resulted in a numerically greater but not statistically significant percentage reduction in the primary endpoint, normalized TAV $(4.8 \%$ and $3.1 \%$ reduction; $\mathrm{P}>0.05$ vs. $\mathrm{SoC})$. No significant differences were observed in absolute 
PAV change and other prespecified secondary efficacy endpoints related to plaque volume, except for absolute change in TAV (nominal $\mathrm{P}=0.02$ vs. SoC).

The lack of a statistically significant difference in the primary efficacy endpoint between the alirocumab and SoC groups needs to be considered in light of several specific factors of the study design, such as the limited sample size $(n=206)$ and the short duration of treatment period $(36$ weeks) derived from a power calculation utilizing 2 previous Japanese IVUS studies (ZEUS and PRECISE-IVUS). These, together with other limiting factors that are described next, may have affected the study's ability to detect a statistically significant change in TAV. ${ }^{9}$

There were important differences in design between J-IVUS, ZEUS, and PRECISE-IVUS. J-IVUS, consistent with other global IVUS studies, included patients with analyzable IVUS images regardless of the existence of plaque and at a longer vessel length (mean vessel length of $18-20 \mathrm{~mm}$ in J-IVUS and minimum of $30 \mathrm{~mm}$ in the global IVUS studies), ${ }^{16,17}$ whereas ZEUS and PRECISE-IVUS analyzed shorter vessel lengths $(8-10 \mathrm{~mm}$ and $10-12 \mathrm{~mm}$, respectively) only targeting part of the vessel with plaques. This led to a smaller proportion of TAV in J-IVUS actually representing the atherosclerotic plaque volume, resulting in a smaller reduction in plaque volume in comparison with the ZEUS and PRECISE-IVUS studies.

The ZEUS study, an open-label study of 24 weeks in 95 patients, demonstrated a difference in TAV reduction of $5.0 \%$ between the atorvastatin/ezetimibe and atorvastatinonly group $(\mathrm{P}=0.06)$, with a difference in LDL-C decrease from baseline of $0.40 \mathrm{mmol} / \mathrm{L}(15.4 \mathrm{mg} / \mathrm{dL})$ between the 2 groups (note PAV was not reported in ZEUS). ${ }^{14}$ The randomized, open-label PRECISE-IVUS trial of 9-12 months conducted in 202 patients demonstrated reduction of TAV by $8.9 \%$ and PAV by $1.5 \%$ between the atorvastatin/ ezetimibe and atorvastatin-only groups, with a difference in LDL-C decrease from baseline of $0.30 \mathrm{mmol} / \mathrm{L}$ $(11.6 \mathrm{mg} / \mathrm{dL})$ between ACS patients in the 2 groups. ${ }^{15}$

In contrast to previously reported IVUS studies, ZEUS and PRECISE-IVUS described large plaque reductions in relation to the observed LDL-C decreases $(5.0-8.9 \%$ TAV reduction and $1.5 \%$ reduction in PAV with LDL-C decreases from baseline of $0.30-0.40 \mathrm{mmol} / \mathrm{L}$ [11.6$15.4 \mathrm{mg} / \mathrm{dL}])$, and over relatively shorter treatment periods of 6-12 months.

The 104-week SATURN study reported a difference in PAV reduction of $-0.2 \%$ between atorvastatin and rosuvastatin groups $(\mathrm{P}=0.17$; percent change in TAV not reported), with a difference in LDL-C decrease from baseline of $0.20 \mathrm{mmol} / \mathrm{L}(7.7 \mathrm{mg} / \mathrm{dL})$ between the 2 groups. ${ }^{18}$ The multinational, randomized, double-blind, placebo-controlled GLAGOV study compared the effect of the PCSK9 inhibitor evolocumab $(420 \mathrm{mg}$ every 4 weeks on top of statin) on plaque progression over a study period of 76 weeks (vs. only 36 weeks in J-IVUS) in 968 patients ( $\mathrm{n}=206$ in J-IVUS) who had a clinical indication for coronary angiography. The primary endpoint reached statistical significance with a $-1.0 \%$ PAV difference vs. placebo when the LDL-C decrease from baseline vs. placebo was $1.46 \mathrm{mmol} / \mathrm{L}(56.5 \mathrm{mg} / \mathrm{dL}$; time-weighted on-treatment value; in J-IVUS the LDL-C decrease from baseline vs. SoC was $1.37 \mathrm{mmol} / \mathrm{L}[52.8 \mathrm{mg} / \mathrm{dL}]$ at Week 12$).{ }^{19} \mathrm{In}$ J-IVUS, the shorter study period ( 9 months) and smaller number of enrolled patients compared with these previous studies may have limited the ability to show a significant difference in plaque volume. Therefore, the present study does not definitively establish alirocumab's efficacy on plaque regression.

Another limiting factor in the analysis of the present study results includes the open-label study design and the ability of investigators to alter background LLT in the SoC arm during the observation period. An increase in ezetimibe therapy occurred in the SoC group, from $7.9 \%$ of patients at randomization to $48.0 \%$ during the study, which may have confounded the results.

Clinical benefits of alirocumab have been demonstrated in the ODYSSEY OUTCOMES trial.9 ODYSSEY OUTCOMES was conducted in 18,924 recent ACS patients over a 2.8-year median double-blind follow-up, and showed a significant reduction in a composite of death from coronary artery disease, nonfatal myocardial infarction, fatal or nonfatal ischemic stroke, or unstable angina requiring hospitalization with alirocumab vs. placebo (HR $[95 \% \mathrm{CI}]$ : $0.85[0.78-0.93]) .{ }^{9}$ In a prespecified analysis of the trial, no interaction was observed between treatment effect and geographic regions (interaction P-value not significant: 0.40). ${ }^{9}$ The separation of the MACE Kaplan-Meier curves in the ODYSSEY OUTCOMES study after 1 year of treatment also suggested the need for a longer treatment period than the one used in J-IVUS to observe the full effect of alirocumab.

Alirocumab was generally well tolerated, and the safety data were consistent with previously published safety data regarding alirocumab., ${ }^{9,20}$

\section{Conclusions}

In Japanese patients who had ACS events and whose LDL-C was $\geq 2.59 \mathrm{mmol} / \mathrm{L}(\geq 100 \mathrm{mg} / \mathrm{dL})$ at ACS diagnosis, alirocumab $75 \mathrm{mg}$ Q2 W treatment in addition to stable dose statin therapy with or without other LLTs resulted in a numerically greater percent reduction in normalized TAV vs. SoC, which did not reach statistical significance over 36 weeks of follow-up. Consistent with previously published ODYSSEY results, alirocumab substantially reduced LDL-C levels, with reductions being maintained at all time points up to Week 36. Alirocumab was generally well tolerated and no particular safety concerns were identified.

\section{Acknowledgments}

The authors thank the patients, their families, and all investigators involved in this study. The authors also thank Micron, Inc. (imaging analysis; Kobe, Japan), EPS Corporation (statistical analysis and clinical research operation; Tokyo, Japan), and the following people from the study sponsors for study operation support: Yuko Azuma, Noriyuki Kanda, Naoko Kudo, Takahiro Nakama, and Makiko Usami (Sanofi). The following people from the study sponsors provided critical review of the manuscript: Michael Howard, Asuka Ozaki, and Timothée Sourdille (Sanofi), and Richa Attre, Garen Manvelian, and Robert Pordy (Regeneron Pharmaceuticals, Inc.). A full list of principal investigators and committee members is shown in the Supplementary File. Medical writing assistance and editorial support was provided by Susanne Ulm, PhD, of Prime (Knutsford, UK), supported by Sanofi and Regeneron Pharmaceuticals, Inc. according to Good Publication Practice guidelines (https://annals. org/aim/fullarticle/2424869/good-publication-practice-communicat ing-company-sponsored-medical-research-gpp3). The sponsor was involved in the study design and the collection, analysis, and interpretation of data, as well as data checking of information provided in the manuscript. The authors were responsible for all content and editorial decisions, and received no honoraria related to the development of this publication. 


\section{Sources of Funding}

This study was funded by Sanofi and Regeneron Pharmaceuticals, Inc.

\section{Disclosures}

Consultancy

Michael J Louie: employee of Regeneron Pharmaceuticals, Inc.

Kiyoko Uno and Yoshiharu Takagi: employees of Sanofi.

Stock/Stock Options

Michael J Louie: Regeneron Pharmaceuticals, Inc.

Kiyoko Uno: Sanofi K.K.

\section{Remuneration}

Junya Ako: Sanofi.

Kenichi Tsujita: Bayer Yakuhin, Ltd., Daiichi Sankyo Co., Ltd., Kowa Pharmaceutical Co. Ltd., MSD K.K., Sanofi K.K., Takeda Pharmaceutical Co., Ltd.

Takafumi Hiro: Bayer Yakuhin, Ltd.

Yoshihiro Morino: Sanofi Aventis.

Toshiro Shinke: Abbott Vascular, Dai-ichi Sankyo, Bayer, Sanofi, Nipro.

Hiromasa Otake: Sanofi, Terumo, Micron.

Research Funding

Kenichi Tsujita: AstraZeneca K.K., Sugi Bee Garden, Japan Medical Device Technology Co., Ltd.

Scholarship Funds or Donations

Kenichi Tsujita: ITI Co., Ltd., Astellas Pharma Inc., Abbott Vascular Japan Co., Ltd., Otsuka Pharmaceutical Co., Ltd., Kaneka Medix Co., Ltd., Goodman Co., Ltd., GM Medical Co., Ltd., Daiichi Sankyo Co., Ltd., Takeda Pharmaceutical Co., Ltd., Mitsubishi Tanabe Pharma, Chugai Pharmaceutical Co, Ltd., TERUMO Co., Ltd., Boehringer Ingelheim Japan, Medtronic Japan Co., Ltd., Japan Lifeline Co., Ltd., Novartis Pharma K.K., Fides-one, Inc., BristolMyers K.K., Boston Scientific Japan K.K., Cardinal Health Japan, MSD K.K.

Hiromasa Otake: Boston Scientific, Sanofi.

Endowed Departments by Commercial Entities

Takafumi Hiro: This author also works for a department endowed by Boston-Scientific Japan Co, Ltd. at Nihon University School of Medicine.

\section{References}

1. Pilgrim T, Vranckx P, Valgimigli M, Stefanini GG, Piccolo R, Rat $\mathrm{J}$, et al. Risk and timing of recurrent ischemic events among patients with stable ischemic heart disease, non-ST-segment elevation acute coronary syndrome, and ST-segment elevation myocardial infarction. Am Heart J 2016; 175: 56-65.

2. Jernberg T, Hasvold P, Henriksson M, Hjelm H, Thuresson M, Janzon M. Cardiovascular risk in post-myocardial infarction patients: Nationwide real world data demonstrate the importance of a long-term perspective. Eur Heart J 2015; 36: 1163-1170.

3. Anderson JL, Adams CD, Antman EM, Bridges CR, Califf RM, Casey DE Jr, et al. 2012 ACCF/AHA focused update incorporated into the ACCF/AHA 2007 guidelines for the management of patients with unstable angina/non-ST-elevation myocardial infarction: A report of the American College of Cardiology Foundation/American Heart Association Task Force on Practice Guidelines. J Am Coll Cardiol 2013; 61: e179-e347.

4. Amsterdam EA, Wenger NK, Brindis RG, Casey DE, Ganiats TG, Holmes DR, et al. 2014 AHA/ACC guideline for the management of patients with non-ST-elevation acute coronary syndromes. J Am Coll Cardiol 2014; 64: e139.

5. Jneid H, Addison D, Bhatt DL, Fonarow GC, Gokak S, Grady KL, et al. 2017 AHA/ACC clinical performance and quality measures for adults with ST-elevation and non-ST-elevation myocardial infarction: A Report of the American College of Cardiology/American Heart Association Task Force on Performance Measures. J Am Coll Cardiol 2017; 70: 2048-2090.

6. Teramoto T, Sasaki J, Ishibashi S, Birou S, Daida H, Dohi S, et al. Executive summary of the Japan Atherosclerosis Society (JAS) guidelines for the diagnosis and prevention of atherosclerotic cardiovascular diseases in Japan: 2012 version. $J$ Atheroscler Thromb 2013; 20: 517-523.

7. Catapano AL, Graham I, De Backer G, Wiklund O, Chapman MJ, Drexel H, et al. 2016 ESC/EAS Guidelines for the Management of Dyslipidaemias. Eur Heart J 2016; 37: 2999-3058.

8. Grundy SM, Stone NJ, Bailey AL, Beam C, Birtcher KK, Blumenthal RS, et al. 2018 AHA/ACC/AACVPR/AAPA/ABC/ ACPM/ADA/AGS/APhA/ASPC/NLA/PCNA Guideline on the Management of Blood Cholesterol: A Report of the American College of Cardiology/American Heart Association Task Force on Clinical Practice Guidelines. J Am Coll Cardiol 2019; 73: $3168-3209$

9. Schwartz GG, Steg PG, Szarek M, Bhatt DL, Bittner VA, Diaz $\mathrm{R}$, et al. Alirocumab and cardiovascular outcomes after acute coronary syndrome. $N$ Engl J Med 2018; 379: $2097-2107$.

10. Mintz GS, Nissen SE, Anderson WD, Bailey SR, Erbel R, Fitzgerald PJ, et al. American College of Cardiology clinical expert consensus document on standards for acquisition, measurement and reporting of intravascular ultrasound studies (IVUS): A report of the American College of Cardiology Task Force on Clinical Expert Consensus Documents. J Am Coll Cardiol 2001; 37: $1478-1492$.

11. Nicholls SJ, Hsu A, Wolski K, Hu B, Bayturan O, Lavoie A, et al. Intravascular ultrasound-derived measures of coronary atherosclerotic plaque burden and clinical outcome. $\mathrm{J} \mathrm{Am} \mathrm{Coll}$ Cardiol 2010; 55: 2399-2407.

12. Ako J, Hibi K, Kozuma K, Miyauchi K, Morino Y, Shinke T, et al. Effect of alirocumab on coronary atheroma volume in Japanese patients with acute coronary syndromes and hypercholesterolemia not adequately controlled with statins: ODYSSEY J-IVUS rationale and design. $J$ Cardiol 2018; 71: 583-589.

13. Friedewald WT, Levy RI, Fredrickson DS. Estimation of the concentration of low-density lipoprotein cholesterol in plasma, without use of the preparative ultracentrifuge. Clin Chem 1972; 18: $499-502$.

14. Nakajima N, Miyauchi K, Yokoyama T, Ogita M, Miyazaki T, Tamura $\mathrm{H}$, et al. Effect of combination of ezetimibe and a statin on coronary plaque regression in patients with acute coronary syndrome: ZEUS trial (eZEtimibe Ultrasound Study). IJC Metabol Endocr 2014; 3: 8-13.

15. Tsujita K, Sugiyama S, Sumida H, Shimomura H, Yamashita T, Yamanaga K, et al. Impact of dual lipid-lowering strategy with ezetimibe and atorvastatin on coronary plaque regression in patients with percutaneous coronary intervention: The multicenter randomized controlled PRECISE-IVUS trial. J Am Coll Cardiol 2015; 66: 495-507.

16. Nissen SE, Tuzcu EM, Brewer HB, Sipahi I, Nicholls SJ, Ganz P, et al. Effect of ACAT inhibition on the progression of coronary atherosclerosis. $N$ Engl J Med 2006; 354: 1253-1263.

17. Nissen SE, Tuzcu EM, Schoenhagen P, Brown BG, Ganz P, Vogel RA, et al. Effect of intensive compared with moderate lipid-lowering therapy on progression of coronary atherosclerosis: A randomized controlled trial. JAMA 2004; 291: 1071-1080.

18. Nicholls SJ, Ballantyne CM, Barter PJ, Chapman MJ, Erbel $\mathrm{RM}$, Libby $\mathrm{P}$, et al. Effect of two intensive statin regimens on progression of coronary disease. N Engl J Med 2011; 365: 2078 2087.

19. Nicholls SJ, Puri R, Anderson T, Ballantyne CM, Cho L, Kastelein JJ, et al. Effect of evolocumab on progression of coronary disease in statin-treated patients: The GLAGOV randomized clinical trial. JAMA 2016; 316: 2373-2384.

20. Jones PH, Bays HE, Chaudhari U, Pordy R, Lorenzato C, Miller $\mathrm{K}$, et al. Safety of alirocumab (a PCSK9 monoclonal antibody) from 14 randomized trials. Am J Cardiol 2016; 118: 1805-1811.

\section{Supplementary Files}

Please find supplementary file(s);

http://dx.doi.org/10.1253/circj.CJ-19-0412 